\title{
Geduld und Ungeduld. Komplementäre Haltungen in der (zeitbegrenzten) institutionellen psychoanalytischen Psychotherapie
}

\author{
Daniel Sollberger (Liestal)
}

Zusammenfassung: Die stationäre wie auch teilstationäre Psychotherapie steht unter dem Verdikt der Zeitbegrenzung. Eine klassische Voraussetzung für psychoanalytische Therapien, wie sie in der Grundregel der freien Assoziation zum Ausdruck kommt, ist die frei gestaltbare Zeit. Konsequenz dieser Zeitbegrenzung ist die Fokussierung, das heisst die Selektion und damit Einschränkung der freien Assoziation. Dies wurde mit der Entwicklung von psychodynamischen Fokal- und Kurztherapien breit diskutiert. Dennoch halten psychoanalytische Verfahren daran fest, das Material der Bearbeitung zum einen im Hier und Jetzt entstehen zu lassen, was auf Seite der Patienten der Methode der freien Assoziation entspricht. Zum andern soll eine Fokussierung auf die Subjektivität der Patienten erfolgen, der auf Seiten der Therapeuten die gleichschwebende Aufmerksamkeit entspricht. Hier eröffnet sich ein Konflikt, der sich in der psychotherapeutischen Haltung manifestiert, wenn es darum geht, Therapieprozessen im Längsverlauf mit Geduld zu begegnen, umgekehrt aber in den Einzelsitzungen durchaus die Ungeduld in der Gegenübertragung zu reflektieren. Sie soll nutzbar gemacht werden, um der Tendenz von Therapeut und Patient entgegenzutreten, aufgrund einer rationalen und bewussten Fokussierung aggressive, selbstdestruktive, ein Leben ausserhalb der Therapie sabotierende Verhaltensweisen als Thema zu vermeiden oder gar zu ignorieren. Beispielhaft wird die Übertragungsfokussierte Therapie (TFP) als Modifikation psychoanalytischer Psychotherapie und Möglichkeit institutionellen Arbeitens in ihrem Bestreben dargestellt, dem Paradox von gleichschwebender Aufmerksamkeit in der Hier-und-Jetzt-Situation der Therapie und der besonderen Aufmerksamkeit auf die wesentlichen äusseren Bereiche des Lebens von Patienten zu begegnen.

Schlüsselwörter: psychodynamische Psychotherapie, übertragungsfokussierte Psychotherapie, therapieexterne Realität, Übertragung, Gegenübertragung 


\section{Einleitung: Therapeutische Beziehung und psychoanalytische Haltung}

Im Zentrum jeder Therapie steht die Beziehung zwischen Patient und Therapeut. ${ }^{1}$ Allerdings wird diese unterschiedlich reflektiert, konzeptualisiert und eingesetzt. In ihrer breit angelegten metaanalytischen Psychotherapiestudie, «Die grosse Psychotherapie-Debatte» (Wampold, Imel et al., 2018), haben die beiden renommierten Psychotherapieforscher Bruce E. Wampold und Zac E. Imel unzählige empirische Psychotherapiestudien auf ihre sogenannten Hintergrundmodelle hin untersucht. Sie kontrastieren die empirischen Befunde dabei in zwei Modellen, die sie in der Forschung als zwar leitend, allerdings nicht explizit ausgewiesen erkennen.

Das «medizinische Metamodell» (MM) zeichnet sich dadurch aus, dass Symptome als Zeichen einer zugrundeliegenden Störung verstanden und taxonomisch klassifiziert werden können und die Störung durch eine psychologische Hypothese erklärt wird. Der Veränderungsmechanismus wird dabei einem spezifischen psychotherapeutischen Verfahren zugeschrieben, so dass letztlich ein spezifischer Faktor identifiziert ist, der speziell für die vorliegende Störung als wirksam gilt.

Im Gegensatz dazu geht das «kontextuelle Metamodell» (KM) davon aus, dass Psychotherapie eine emotional hochbesetzte Beziehungsform ist, die eine hilfesuchende Person vertrauensvoll mit einem professionellen Therapeuten eingeht. Die hilfesuchende Person glaubt - und diese Überzeugung wird von Therapeutenseite unterstützt -, dass der Therapeut tatsächlich hilfreich handelt und dass es Pläne oder Narrative gibt, die eine plausible Erklärung für die Probleme liefern und ein «Ritual» oder Prozedere bereitstellen, um diese zu bewältigen.

Während das MM annimmt, dass die besseren Therapieerfolge auf eine bestimmte, spezifische Technik zurückzuführen sind, macht das KM dieVorhersage, dass sich keine spezifischen Effekte erkennen lassen, sondern nur generelle; sie liefern die besseren Effekte als die an spezifische Techniken gebundene.

Bereits M. J. Lambert hat in seinen Psychotherapie-Outcome-Forschungen nachgewiesen, dass der Faktor «therapeutische Beziehung» einen gegenüber spezifischen Methoden doppelt so grossen Einfluss auf die Therapieresultate hat, nämlich etwa zu einem Drittel daran beteiligt ist, während methodenspezifische und Erwartungseffekte je nur 15\% ausmachen und der Hauptteil bei extratherapeutischen Veränderungen liegt (Lambert, 1992).

In dieselbe Richtung geht die Wampold/Imel-Studie: Alle Psychotherapien sind erfolgreich (Dodo-Bird-Effekt), die durchschnittlichen Effektstärken aller Verfahren liegen im selben Bereich. ${ }^{2}$ Die Unterschiede zwischen den verschiede- 
nen Therapieverfahren scheinen weit weniger gross zu sein als jene zwischen den Therapeuten derselben Therapieschule. Auch haben jene Therapeuten, die nicht auf Technik, sondern Kontakt setzen, die besseren Erfolge. Worauf es vor allem anzukommen scheint, ist die Fähigkeit von Therapeutinnen und Therapeuten, sich nicht verwickeln zu lassen und aggressive Gegenübertragungsreaktionen bei schwierigen Patienten zu containen.

Die Etablierung und systematische Reflexion der therapeutischen Beziehung kommt in psychodynamischen Therapien nicht nur im Sinn einer spezifischen Technik als vor allen Dingen als therapeutische Haltung zum Tragen. Der Stellenwert einer solchen Haltung bildet sich denn auch in den psychoanalytischen Ausbildungscurricula in Selbsterfahrung, Supervision, Fallkonzeptionen u.a.m. ab. Man könnte aus derWampold/Imel-Studie folgern, dass sie psychodynamische Denkweisen in der Medizin stärkt, Denkweisen, die nicht auf eine Medizinalisierung der Psychotherapie, sondern eher umgekehrt auf eine psychodynamisch reflektierte Haltung in der Medizin abzielen. Um es vereinfacht auf den Punkt zu bringen: eine psychodynamische Haltung qua beziehungsfokussierte und -reflektierte Gestaltung und Nutzung der therapeutischen Beziehung, nicht störungsspezifische Technik führen zum Erfolg.

\section{Veränderungen in der psychoanalytischen Psychotherapie und im institutionellen Umfeld}

Richtet man den Blick auf die institutionelle Psychiatrie, in welcher die in den Ausbildungscurricula erworbenen Kenntnisse und Methoden ihre direkte klinische und alltägliche Anwendung und Einübung finden, dann stellt sich die Frage, in welcher Form und inwieweit dort die angesprochene psychodynamische Haltung gelebt, vertreten und vermittelt wird. Ich meine, dass Anpassungen von zwei Seiten her erfolgt sind: zum einen betreffen sie Veränderungen der psychoanalytischen Prinzipien selbst, zum andern jene, die seitens der institutionellen psychiatrischen Versorgung gefordert sind.

\subsection{Entwicklungen vom «Gold» zum «Kupfer»}

Die Modifikationen und Weiterentwicklungen der psychoanalytischen Psychotherapie sind vielfältig und längst nicht mehr im Kontext des Reinheitsideals der Psychoanalyse geführt. Das «reine Gold der Analyse» wurde schon zu Zeiten des Gründers in der Massenanwendung dieser Therapie in der «direkten Suggestion» vielfältig abgekupfert (Freud, 1919, 193). Die Modifikationen und Weiterentwicklungen der psychoanalytischen Behandlungsmethoden sind dabei 
der psychoanalytischen Theorieentwicklung durchaus inhärent. Paradigmatisch zeigt sich dies an der viel diskutierten intersubjektiven Wende in der Psychoanalyse, welche sich exemplarisch und zentral am Begriffspaar der Übertragung und Gegenübertragung nachzuzeichnen lässt.

Als «stärkste Waffe des Widerstands», nämlich sich nicht an Verdrängtes erinnern zu müssen, wird die Übertragung für Freud «zum besten Instrument der analytischen Kur» (Freud, 1925d, S. 68f.). Sobald die Deutung der Übertragung die Erinnerung wieder ermöglicht, kann sie aufgelöst werden. Erinnerung statt Wiederholung ist das Ziel. Allerdings wird bei Freud auch die reale interpersonale Bedeutung der Übertragung deutlich, wenn er schreibt, dass die Übertragungsphänomene dazu führen, dass sie «die verbogenen und vergessenen Liebesregungen des Kranken aktuell und manifest machen, denn schliesslich kann niemand in absentia oder in effigie erschlagen werden.» (Freud, 1912b, S. 374). So scheint sich bereits bei ihm ein interpersonales Erfahrungsfeld zwischen Patient und Analytiker zu öffnen, auf welchem gekämpft, zumindest agiert wird und welches beide Akteure verbindet.

Man kann, wie Werner Bohleber dies überzeugend getan hat, die Geschichte der Konzepte von Übertragung und Gegenübertragung anhand der Freudschen Metaphern des Spiegels und des Senders bzw. Receiver des Telefons in zwei Traditionssträngen darstellen und dabei den intersubjektiven Paradigmenwechsel nachvollziehen (vgl. Bohleber, 2018).

Mit der Spiegelmetapher wird der Einfluss des Analytikers auf die Übertragung des Patienten minimiert. Der Analytiker muss zusehen, dass er in Eigenanalyse den Spiegel reinigt und für den Analysanden sauber hält. Diese Vorstellung hat sich deutlich gewandelt. Im Blick auf die interaktive Wechselseitigkeit des analytischen Prozesses wird der Objektivitätsanspruch und die Autorität des Analytikers als Experte in der relationalen Psychoanalyse schliesslich ganz verabschiedet zugunsten der unhintergehbaren Subjektivität der am analytischen Prozess Teilhabenden. Die Übertragungsanalyse zielt nicht mehr auf die Erinnerung der Vergangenheit ab, ganz im Gegenteil könnte man sagen: Wo einst die Übertragung als Widerstand gegen die Erinnerung galt, gilt die Erinnerung jetzt vielmehr als Widerstand gegen die Übertragung (vgl. Altmeyer \& Thomä, 2006, S. 24).

Ausgangspunkt für eine neue Begrifflichkeit eines intersubjektiven Erfahrungsfelds findet sich in Freuds Receiver-Metapher, mit welcher letztlich der Begriff der Gegenübertragung verbunden ist. Paula Heimann hat hier einen «Markstein» (Sandler 1976) gesetzt, indem sie die unmittelbare Reaktion des Analytikers auf den Patienten als Orientierungspunkt für ein Verständnis der unbewussten Prozesse 
des Patienten nimmt. Heimanns Überlegungen gipfeln in der Erkenntnis, dass «die Gegenübertragung des Analytikers nicht nur das A und O der analytischen Beziehung» sei, sondern recht eigentlich «die Schöpfung (creation) des Patienten. Sie ist ein Teil der Persönlichkeit des Patienten» (Heimann, 1950, S. 83).

Mit dem Kleinianischen Konzept der projektiven Identifizierung wurde ein Modell entwickelt, wonach unbewusst unerträgliche Selbstanteile projektiv im Anderen untergebracht und dort kontrolliert werden - ein Modell, welchem W. Bion in der Ausweitung zu einer generellen Form non-verbaler Kommunikation in seiner «Container-contained»-Konzeption den pathologischen Nimbus genommen hat.

Dieses letztlich an Hegels philosophischer Intersubjektivitätstheorie und dem im Herr-Knecht-Verhältnis dargelegten Prozess gegenseitiger Anerkennung orientierte psychoanalytische Konzept führt dazu, dass sich subjektive, psychische Veränderungen im analytischen Prozess detailliert als Veränderungen im interpersonalen Erfahrungsraum nachzeichnen lassen (vgl. Bohleber 2018, S. 719 f.).

Die Qualität der therapeutischen Beziehung ist entscheidend für das Outcome der Behandlungen. Wie keine andere psychotherapeutische Richtung charakterisiert sich die psychoanalytische Theorie dadurch, dass sie die Art und Weise der therapeutischen Beziehung systematisch und vertieft reflektiert. Ob es nun die Übertragungsbeziehung oder schlicht der interpersonelle Verstehens- und Anerkennungsprozess ist, in der Ko-Konstruktion des therapeutischen Feldes, welche nicht allein mit Worten, sondern ebenso mit dem Körper über unbewusste Phantasien von Therapeut und Patient erfolgt, wird die biografische Arbeit zurückgedrängt zugunsten der Unmittelbarkeit der Erfahrung. Der psychoanalytischen Theorie von Psychotherapie liegt somit nicht bloss eine Theorie des Subjekts zugrunde, sondern recht eigentlich eine kritische Theorie der Intersubjektivität. Es steht also in Frage, inwieweit Therapeuten nicht doch in einerWeise Einfluss auf den Therapieverlauf nehmen, indem sie in ihrer Ko-Konstruktion des ÜbertragungsGegenübertragungsgeschehens letztlich eigene, unbewusste Anteile agieren und damit im Sinne Freuds in «direkter Suggestion» auf den Patienten - mehr oder weniger - supportiv einwirken, nicht also, wie Freud oder später Bion es fordern, voraussetzungs- und absichtslos in «gleichschwebender Aufmerksamkeit», «ohne Erinnerung oder Begehren» technisch neutral bleiben.

\subsection{Veränderungen im institutionellen Kontext}

Neben diesen Veränderungen innerhalb der psychoanalytischen Theoriebildung und ihren Auswirkungen auf die Behandlungsmodi änderten in der psychiatrischen und psychotherapeutischen Versorgung die Bedingungen zum einen hin 
zu einer besseren Versorgung breiter Bevölkerungskreise und Anspruchsgruppen. Zum andern entstand über die letzten Jahrzehnte ein zunehmender Kosten- und Finanzierungsdruck im Gesundheitswesen. Die Forderung nach effektiven, zweckmässigen und insbesondere wirtschaftlichen Behandlungsformen wurde immer lauter. Auch psychodynamische Psychotherapieverfahren haben sich dieser Herausforderung in kreativer Weise gestellt und Konzepte der Kurz- und Fokaltherapien entwickelt, die eine Modifikation der Grundprinzipien psychoanalytischer Behandlungen erforderte (vgl. Küchenhoff, 2005). Im Zentrum stehen die Zeitbegrenzung und der daraus potentiell erwachsende Konflikt zwischen der notwendigen Fokussierung der therapeutischen Arbeit und den psychoanalytischen Grundprinzipien der freien Assoziation und gleichschwebenden Aufmerksamkeit. Wie passt das zusammen, einerseits einen angesichts der begrenzten Zeit vordefinierten Therapiefokus festzulegen, andererseits Patienten dennoch dazu anzuhalten, ihre Gedanken und Einfälle möglichst unzensuriert, assoziativ und in offener Form zur Sprache zu bringen, nicht also an einem bestimmten Fokus oder Ziel orientiert sich zu sehr zu verschliessen?

Wenn psychoanalytische Therapieverfahren sich gegenüber anderen Verfahren in ihrer prinzipiellen Haltung dadurch auszeichnen, dass bei ihnen der Gegenstand der Klärungs- und Deutungsarbeit der analytischen Therapie nicht vorausgeht, sondern sich in der Übertragungsbeziehung oder vielleicht noch allgemeiner: der interpersonellen Beziehung erst entfaltet, wie lässt sich diese Haltung angesichts der Anforderungen verkürzter Therapiedauer aufrechterhalten? Wenn das affektiv bedeutsame Material erst aus der therapeutischen Beziehung hic et nunc emergiert, wie lässt sich dies mit einem anfangs der Therapie festgelegten Therapiefokus vereinbaren? Wenn nicht etwas, das schon bewusst und sprachlich fassbar wäre, am Anfang der Therapie steht, sondern Patienten in ihren (auch) unbewussten Bedürfnissen, Wünschen, Befürchtungen und Ängsten das Gegenüber brauchen, um zusammen mit ihm eine Szene zu schaffen, in und aus welcher letztere erst zu Bewusstsein und zur Sprache kommen können, wie verträgt sich dies mit derVerankerung der Therapie in einer aussertherapeutischen Realität?

Zeitbegrenzung und die daraus resultierende Fokalität der zu bearbeitenden Themen einer psychoanalytischen Psychotherapie geraten nicht nur hinsichtlich der freien Assoziation und der gleichschwebenden Aufmerksamkeit potentiell in einen Konflikt, sondern leisten insbesondere dem Verlassen einer technischen Neutralität Vorschub, indem sie Therapeuten zu überfürsorglichen 
Haltungen verführen und damit potentiell in eine Verantwortungsdiffusion. Das Risiko besteht, das Therapeuten aufgrund des Zeitdrucks und des Wunsches oder der Selbstverpflichtung, therapeutisch möglichst erfolgreich zu sein, eigene Befürchtungen oder auch die eigene Ungeduld unreflektiert agieren. Die Empfehlung oder der Ratschlag gegenüber einem Patienten dienen dann möglicherweise ebenso sehr der eigenen Beruhigung der Therapeuten, etwa weil die Verstörung, Unsicherheit und Angst oder Aggression des Patienten kaum auszuhalten sind. Ein Ratschlag ist dann nicht mehr als einfach nur «gut gemeint» zu verstehen, sondern adressiert implizit zugleich die Botschaft an den Patienten, dass sein emotionaler Zustand doch möglichst kontrolliert bleiben sollte. Ebenso könnte angesichts der verzwickten und komplexen psychosozialen Situation eines Patienten eine supportive Haltung auch als Agieren der Ungeduld des Therapeuten verstanden werden.

Im Fall von exemplarisch kurzen Kriseninterventionen, in denen rasche Hilfestellungen und Halt angeboten werden, zeigen sich durchaus auch komplexe Übertragungs-, Gegenübertragungs- und projektiv-identifikatorische Prozesse. Sie können bspw. dazu führen, dass bei einer Krise aufgrund einer Partnerschaftstrennung beim verlassenen Partner sehr aggressive und kaum erträgliche Affekte entstehen. In einer Krisenintervention würden diese möglicherweise auf den helfenden Therapeuten übertragen, so dass dieser zu spüren bekommt, was der Patient nicht aushält, insbesondere dann, wenn letzterer plötzlich die therapeutische Beziehung abbricht und der Therapeut «verlassen» zurückbleibt. Umgekehrt bergen auch positive, idealisierende Übertragungsmechanismen in Kriseninterventionen das Risiko, dass die Behandlung nur unter grosser Kränkung des Patienten beendet werden kann und damit die Intervention insgesamt erschweren.

Auch in der Krisenintervention gilt es die richtige Balance zu finden zwischen einer notwendigen äusseren Entlastung, einer realen Veränderung und inneren Stabilisierung auf der einen Seite und «dem Standhalten, Erkennen und Verstehen des Übertragungsgeschehens und der diesem zugrunde liegenden inneren Dynamik auf der anderen Seite» (Till, 2009, S. 786).

Wenn also der Anspruch aufrechterhalten bleiben soll, auch angesichts der komplexen interpersonellen Übertragungsdynamiken mit Infragestellung einer technischen Neutralität (s. 2.1) wie auch angesichts der Zeitverkürzungen und daraus resultierender Fokussierung von Themen in der Therapie (s. 2.2) nicht «suggestiv», supportiv oder pädagogisch zu werden, sondern nach psychoanalytischen Prinzipien zu verfahren unter Beibehaltung der technischen Neutralität und der 
Arbeit in und an der Beziehung mit der Aufforderung an die Patienten möglichst frei zu assoziieren und an die Therapeuten, jede Sitzung in gleichschwebender Aufmerksamkeit zu verfolgen, dann bedarf es einer näheren Klärung, wie mit diesem Dilemma in der therapeutischen Beziehung umzugehen ist.

\section{Strukturierung des Dilemmas in der Übertragungsfokussierten Therapie (TFP)}

Paradigmatisch kann die von Otto F. Kernberg und seiner Forschungsgruppe entwickelte übertragungsfokussierte Therapie (Transference Focused Psychotherapy TFP) als strukturierte psychoanalytische Antwort auf das dargelegte Dilemma verstanden werden - ein Verfahren nota bene, das gerade in institutionellen Kontexten, in welchen psychiatrische Exploration, sozialpsychiatrische Interventionen, Zeitbegrenzungen u.a.m. eine zentrale Rolle spielen, erlaubt, psychodynamische Denkweisen und eine psychoanalytische Haltung aufrechtzuerhalten.

Die TFP wurde explizit für Patienten entwickelt, deren Krankheitsschweregrad und die Dringlichkeit ihrer problematischen Lebenssituation eine Therapie erfordern, die zugleich aber grösste Schwierigkeit haben, einen Therapierahmen aufrechtzuerhalten. So dass viele Therapeuten supportive Behandlungen bei diesen Patienten als indiziert erachten. Demgegenüber hat die TFP den Anspruch, über eine symptomreduzierende Behandlung hinaus - also etwa einer Reduktion von chronisch suizidalem oder antisozialem Verhalten, Substanzmissbrauch oder Essstörungen - eine Veränderung in der Persönlichkeitsstruktur, i.e. der Identitätsdiffusion als Grundmerkmal der Borderline-Organisation und in der Folge des sozialen Funktionsniveaus in Arbeit und Beruf sowie Freundschafts- und intimen Beziehungen dieser Patienten zu erwirken.

\subsection{Das Modell der Übertragungsfokussierten Therapie}

Die TFP unterscheidet allgemeine und übergeordnete Behandlungsziele im Sinn von «strategischen Prinzipien», die Systematisierung von Interventionen in Einzelsitzungen als «Taktiken» und spezifische psychoanalytische «Techniken» (s. Yeomans, Clarkin et al., 2017).

Unter der Annahme, dass Patienten mit schweren strukturellen Störungen im Kern am Syndrom einer Identitätsdiffusion leiden, zielen die strategischen Prinzipien der TFP auf die Arbeit an einer Integration von abgespaltenen internalisierten Selbst- und Objektrepräsentanzen, wie sie in der therapeutischen Übertragungsbeziehung aktiviert werden und zum Ausdruck kommen. Konkret 
erfolgt diese Arbeit gemäss psychoanalytischen Prinzipien in der Aufforderung zur freien Assoziation des Patienten und im Zuhören, Beobachten, Identifizieren, Klären, Konfrontieren und schliesslich Deuten der sich in den Therapiesitzungen etablierenden, von positiven, libidinös-idealisierenden oder negativen, aggressivverfolgenden Affekten dominierten und abgewehrten Objektbeziehungsdyaden.

Daran orientiert sich die Gesamttherapie, während davon unterschieden die Taktiken die Orientierungshilfe für das Vorgehen in der jeweiligen Sitzung ergeben. Sie bilden die eigentliche Modifikation psychoanalytischer Technik und umfassen im klaren Festlegen von Regeln u.a. den behandlungstechnischen Rahmen (Sitzungsfrequenz, Umgang mit Absenzen, Vorgehen im Notfall, Verantwortungsklärungen, Grenzen der technischen Neutralität u. Ä.) sowie die Hierarchisierung bedeutsamer und für die Therapie prioritär zu behandelnden Themen (Suizidalität, Fremdgefährdung, Therapieabbruchimpulse, Unehrlichkeit, Vertragsbrüche, Ausweichen auf triviale Themen, Agieren zwischen den Sitzungen usw.).

Gerade in der Festlegung von Therapievereinbarungen findet gegenüber klassisch psychoanalytischer Psychotherapie die Exploration besonders bedrohlicher Lebensumstände einen grossen Stellenwert. Fragen nach potentieller Selbstund Fremdgefährdung, nach Risiken eines Therapieabbruchs werden angemessen und strukturiert geklärt. Ebenso grundlegend in einer Therapievereinbarung sind die Klärung eines potentiellen bzw. manifesten sekundären Krankheitsgewinns. Auch wird antizipatorisch bearbeitet, dass bei Gefährdung des Lebens des Patienten oder der Therapie limitierende und restriktive Massnahmen seitens des Therapeuten erfolgen können, die dann zugleich hinsichtlich ihrer Bedeutung in der Übertragung zu klären sind. Gerade diese Verbindung von Grenzsetzung, ggf. in Verbindung mit vorübergehendem Verlassen einer technischen Neutralität auf der einen und der Deutung auf der anderen Seite ist für die TFP charakteristisch und hat sich als sehr effektiv, ja, oftmals lebensrettend erwiesen. Bezüglich der Hierarchie der Themen bzw. der Auswahl eines Hauptthemas in der einzelnen Sitzung deckt sich das taktische Vorgehen der TFP mit der allgemeinen psychoanalytischen Regel, das Material mit der stärksten affektiven Besetzung zu verfolgen und in der Übertragungsbeziehung zu interpretieren. Behandlungstechnisch wird immer die Übertragung und die äussere Realität des Patienten gleichermassen beachtet und flexibel fokussiert - ein taktisches Vorgehen, das bereits Fenichel empfohlen hatte (Fenichel, 1939/2001, S. 160; vgl. auch Kernberg 2014, S. 8 f.). 


\subsection{Fokus auf die therapieexterne Realität}

Die Anwendung behandlungstechnischer Instrumente, nämlich der Klärung, Konfrontation (im Sinn eines taktvollen Aufgreifens von Widersprüchen und Inkonsistenzen etwa verbaler Inhalte und nonverbaler Mitteilungen), Deutung, Übertragungsanalyse und technischen Neutralität als Haupttechniken, bleibt also eng bezogen auf die therapie(sitzungs)externe Realität des Patienten, um eine Dissoziation von Therapie und Lebensrealität ausserhalb zu vermeiden (vgl. Kernberg, 2016). Das heisst, dass die zu Beginn der Therapie gut explorierten, dominanten Schwierigkeiten im realen Leben des Patienten, die ihn letztlich in die Therapie geführt haben, präsent gehalten und gegebenenfalls auch aktiv vom Therapeuten eingebracht werden, auch wenn - oder gerade wenn - sie in der Übertragungsbeziehung nicht dominant zu sein scheinen. So dass möglicherweise Material, das anzusprechen der Patient vermeidet, gar in Abweichung von der technischen Neutralität insbesondere dann aufgegriffen wird, wenn es in irgendeiner Weise den Patienten oder Dritte und damit die Therapie selbst gefährden könnte, also bspw. schwere, vom Patienten nicht geäusserte suizidale Gedanken, die Massnahmen erfordern, um z. B. Vorbereitungshandlungen für einen Suizid zu unterbinden. Hierbei wird intensiv daran gearbeitet, die technische Neutralität wiederherzustellen, was durch eine konsequente Analyse der Übertragungsimplikationen möglicher «nicht neutraler» Interventionen geschieht. Es wird also detailliert geklärt, was den Patienten dazu geführt hat, den Therapeuten in die Situation zu bringen, aufgrund einer grossen Besorgnis um das Wohl des Patienten seine technische Neutralität zu verlassen und gegebenenfalls sogar gegen den Willen des Patienten zu handeln.

In der therapeutischen Arbeit mit Techniken der Klärung, Konfrontation und Deutung mit Fokus auf das "gegenwärtige Unbewusste» (Sandler \& Sandler 1987) werden die langfristigen Ziele im Blick behalten. Das aktive Einbeziehen der äusseren Realität unterscheidet die TFP auf der einen Seite fundamental von kleinianischen und ich-psychologischen Ansätzen psychoanalytischer Psychotherapie. In Einklang mit A. Green, der in Bezug auf Borderline-Patienten das Springen von einem Thema zum nächsten als Vermeidungsverhalten beschreibt, welches eine «zentrale phobische Position» (Green, 2002) zum Ausdruck bringe, erfolgen auch in der TFP häufige Übertragungsdeutungen, um dem Spaltungsprozess bzw. diesem Vermeiden von «traumatogenen Assoziationen», wie es im abrupten Wechsel von Themen in der freien Assoziation bei diesen Patienten zum Ausdruck kommt, entgegenzuwirken und in der Kontinuität von Deutungen Verbindungen zu schaffen (vgl. Kernberg, 2016). 
Auf der andern Seite erfolgt der Fokus auf die äussere Realität des Patienten im Gegensatz zur mentalisierungsbasierten Therapie (Bateman \& Fonagy, 2004) aber nicht in der Absicht, den Mentalisierungsprozess in Bezug auf diese Realität direkt zu beeinflussen, etwa indem der Patient eine vertiefte Einsicht in seine Motive und jene anderer erhält. Vielmehr steht die Exploration der Bedeutung in der Übertragungsbeziehungim Vordergrund, d.h. die Frage, welche Bedeutung es in der therapeutischen Beziehung hat, dass bspw. ein selbstdestruktives Verhalten in der äusseren Realität aussen verbleibt und nicht in der Therapie thematisiert wird.

Im Blick bleiben soll in der psychoanalytischen Arbeit an der Übertragung unter Berücksichtigung und Zuhilfenahme der Gegenübertragungsreaktionen die äussere Realität des Patienten, d.h. insbesondere die zentralen Lebensbereiche, wie sie Kernberg in Liebe und Sexualität, Arbeit und Beruf, Sozialleben und Kreativität erkennt. Diese vier Bereiche bilden Orientierungspunkte, bei welchen sich Therapeuten - ohne einem hypertrophierten Therapieehrgeiz zu verfallen - im Blick auf Therapieziele und Prognosen fragen sollten, welche Ziele ein Mensch in der Situation des Patienten erreichen könnte, wäre er von den Belastungen der Erkrankung befreit. Es sind denn auch die Bereiche, in welchen Patienten ihre selbstdestruktiven Verhaltensweisen zeigen, die abgespalten von der Therapiesitzung zum Agierfeld werden und bei denen für Therapeuten das Risiko besteht, dass sie durch die Abspaltung, die Dissoziation oder auch das bewusste Vermeiden und Verleugnen von Patienten verführt sind, mitzugehen, sich zu beruhigen und in Sicherheit zu wähnen. Oder aber es droht ein Agieren der Gegenübertragung, indem überfürsorgliche oder harmonisierende Haltungen dort eingenommen werden, wo der Patient Verantwortung für sich und die Therapie übernehmen sollte.

\section{Fallbeispiel:}

Als typisches Beispiel möchte ich jenes einer 22-jährigen, intelligenten jungen Frau mit Maturaabschluss und Möglichkeiten, ein Studium zu beginnen, anführen. Sie verletzt sich über Jahre anhaltend selbst und sorgt immer wieder mit suizidalen Impulsen für viel Aufruhr. In der Therapie sind offene Gespräche über die Schwierigkeiten und die auch unbewussten Motive der Patientin möglich, sie kommt zu vielen Einsichten, allerdings ändert die Patientin in der äusseren Realität kaum etwas. Vielmehr entsteht der Eindruck eines Stillstandes. Dabei nutzt die Patientin unbewusst nahezu jede Gelegenheit, eine Verantwortung für sich und ihr Tun zu umgehen und damit letztlich indirekt anderes und andere für ihren Zustand verantwortlich zu machen und ihnen dies zu spüren zu geben. So kam es, dass sie nach einer kurzen stationären Krisenintervention, bei welcher ihr (entgegen 
Empfehlungen pharmakotherapeutischer Leitlinien) Benzodiazepine verabreicht wurden, derart Druck auf den ambulanten Pharmakotherapeuten machte, dass dieser die Medikation widerwillig vorerst beibehielt, und erst nach weiteren Wochen auf Abbau drängte. Daraufhin klagt die Patientin über Entzugssymptome, die sie dazu bringen würden, dass sie die Kontrolle über ihre Impulse und Affekte verliere. Es kommt wiederholt zu selbstdestruktivem und fremdaggressivem Verhalten. Dieses wird dann - die Patientin gewissermassen in ihrer Eigenverantwortung entlastend - dem Entzugssyndrom und damit indirekt den behandelnden Ärzten während der Krisenintervention attribuiert, so dass sie auch in ihrer betreuten Wohneinrichtung aufgrund der subjektiven Entzugssymptomatik eine Extrabehandlung mit Rückzugsmöglichkeiten erwirkt. Die (überzeichnet) präsentierte Entzugssymptomatik kaschiert die jetzt zum Ausdruck kommenden und agierten, im Selbstbild der Patientin aber abgespaltenen fremdaggressiven Anteile. Die Orientierung und Planung mit real zu vollziehenden Schritten in Richtung einer Berufsausbildung bzw. eines Studiums werden damit (indirekt) immer wieder torpediert. Die Patientin verharrt dabei in einem «Opferstatus», der sich kompliziert, da sie sich diesen selbst vorwirft, um vordergründig andere zu entlasten und ihre Selbstbestrafungstendenzen rationalisierend zu rechtfertigen und in ihrem selbstdestruktiven Verhaltensmodus zu verbleiben. Schliesslich wird auch die Therapie in dieser Weise funktionalisiert. Sie soll einst allen zum Beweis dienen, dass ihre therapeutischen Bemühungen hoffnungslos waren und man soll verstehen und akzeptieren, ja, es vielleicht sogar als richtig erachten, wenn sie sich umbringt. Mit solchen Überlegungen drohen sich die therapeutischen Bemühungen des Behandlers zu pervertieren, indem er sich abmüht, die Patientin dies aber in den Dienst ihrer Suizidabsichten stellt und damit die Verantwortung für die Therapie ganz an den Therapeuten delegiert.

Wird hier eine unreflektierte supportive Haltung eingenommen und die projektive Abwehrdynamik der Patientin nicht bearbeitet, läuft eine solche Haltung Gefahr, dass sie als projektiv-identifikatorisch erwirkte Bemühung des Therapeuten um das Gute im Leben der Patientin, d.h. letztlich in seiner totalen Verantwortungsübernahme von der Patientin in ihm kontrolliert und un- oder vorbewusst letztlich sabotiert und zerstört wird. Die wohlwollenden Unterstützungsangebote, die der Patientin etwa in der betreuten Wohneinrichtung gemacht werden, bleiben wirkungslos und führen zu wiederholten Frustrationen der Betreuungspersonen, so dass die Patientin hier auf Dauer einen Abbruch der Bemühungen provoziert. Eine an dieser Stelle gut gemeinte, geduldige, die äussere Realität der Patientin allerdings aus dem Blick verlierende Haltung in den 
Einzelsitzungen würde reflektiert in eine Ungeduld sich wandeln müssen angesichts der fehlenden Verantwortungsübernahme. Während genau diese Arbeit an einer Verantwortung umgekehrt in der Langzeitperspektive viel Geduld in Anspruch nehmen könnte.

\section{5}

\section{Geduld und Ungeduld}

Solche Dynamiken gilt es in der Therapie zu erkennen und zu bearbeiten. Die in den meisten Fällen nicht bewusst und intentional durchgeführten Versuche von Patienten, ihre Therapeuten durch eine vordergründig kooperative Mitarbeit in den Einzelsitzungen zu beruhigen und therapieexterne selbstdestruktive Schwierigkeiten und Krisen zu ignorieren und unthematisiert zu lassen, gilt es in ihrer Verführungskraft zu erkennen, um nicht vorschnell einer (zu) supportiv-versorgenden Haltung zu verfallen und die eigene Gegenübertragung mit Ratschlägen, Empfehlungen oder Aufforderungen zu bestimmten Verhaltensweisen bzw. zum Unterlassen selbstdestruktiven Verhaltens zu agieren. Dies bedeutet umgekehrt keineswegs, dass Therapeuten eine moralistische Haltung einzunehmen beginnen. Nicht die Über-Ich-Perspektive gegenüber selbstdestruktiven Verhaltensweisen von Patienten ist gefragt, denn vielmehr die (ethisch getragene) Sorge um deren reales Überleben (vgl. Ticho, 1972). Das letztere kann seitens der Patienten durchaus als moralistischer Vorwurf verstanden werden und geschieht insbesondere bei Patienten, die ihre eigenen strengen und strafenden Über-IchAnteile in der Projektion auf andere in diesen bekämpfen. So dass zunächst diese Problematik einer paranoiden Übertragungsentwicklung zu bearbeiten ist, bevor der Patient erkennen kann, dass und worin seine eigene Verantwortung für die selbstsabotierenden und -destruktiven Verhaltensweisen liegt. Die Konfrontation mit diesen Verhaltensweisen erfolgt also nicht in supportiver Absicht im Sinn einer Verantwortungsteilung oder gar -übernahme anstelle des Patienten, sondern vielmehr im Sinn einer Möglichkeit, die Bedeutung solcher destruktiven Verhaltensweisen und der damit verbundenen (vorgeblichen) Gleichgültigkeit und Nachlässigkeit zu klären in Bezug auf den Patienten, insbesondere aber auch in Bezug auf die therapeutische Beziehung.

Dies erfordert von Seiten der Therapeuten nicht nur eine gute Kenntnis der Schwierigkeiten, die den Patienten in Therapie gebracht haben, d.h. eine gute Exploration (auch) der äusseren Realität des Patienten zu Beginn der Therapie wie auch im Verlauf der Behandlung - wie wir dies von einer psychiatrisch soliden, institutionell abgestützten Grundversorgung fordern. Darüber hinaus braucht es eine grosse Aufmerksamkeit auf die von Patienten selbst induzierten Bedrohungen 
ihres Wohlergehens und ihrer Gesundheit und damit auf die potentiellen oder tatsächlichen selbstdestruktiven Verhaltensweisen in jeder Sitzung. Hier ist eine Ungeduld der Therapeuten durchaus angebracht, während komplementär dazu der Prozess der Gesamtbehandlung und die Arbeit an der Analyse derVerhaltensweisen des Patienten in ihrer Bedeutung in der Übertragungsbeziehung ausserordentliche Geduld erfordert (vgl. Kernberg, 2016). Ungeduld in der Einzelsitzung immer auch mit psychiatrischem Blick auf die äussere Realität und Geduld im therapeutischen Langzeitverlauf in der Arbeit an der Übertragungsbeziehung widersprechen sich nicht, sondern können als sich ergänzende therapeutische Haltungen verstanden und umgesetzt werden. Letztlich wird eine Balancierung der Aufmerksamkeit auf therapieexterne Verhältnisse bei gleichzeitiger Offenheit für die in jeder Sitzung neu sich konstellierenden Übertragungen gesucht. Gerade in der institutionellen psychiatrisch-psychotherapeutischen Arbeit, wo häufig Zeitdruck und damit verbunden wenig reflektiertes - da Gegenübertragungsreaktionen agierendes - supportives Verhalten eine Rolle spielen, erweisen sich therapeutische Haltungen und Verfahren als besonders wertvoll, die wie die TFP psychodynamisches Verstehen und geduldiges Arbeiten in der Übertragung bei gleichzeitiger aufmerksamer Ungeduld gegenüber Realitätsvermeidung bzw. -verleugnung aus Abwehr «traumatogener Assoziationen» (Green) ausbalancieren.

\section{Anmerkungen}

1 Um den Text in Berücksichtigung aller Geschlechter und aller Formen geschlechtlicher Identitäten nicht mit Unterstrichen, Schrägstrichen oder Sternchen schwer lesbar zu machen, erlaube ich mir, in diesem Aufsatz die männliche Form für alle zu verwenden und dort explizit zu differenzieren, wo dies angezeigt ist.

2 Die Effektstärken liegen bei 0.8, d.h. ca. 75\% aller Psychotherapie-Patienten profitieren nachweislich und gesichert von der Psychotherapie - allerdings nicht, wenn es keinen Plan gibt und die Therapeuten nicht selbst an ihre Therapie glauben, s. Wampold \& Imel, 2018, S. $323 \mathrm{ff}$.

\section{Literatur}

Altmeyer, M., Thomä, H. (2006). Einführung: Psychoanalyse und Intersubjektivität. In: dies. (Hrsg), Die vernetzte Seele. Die intersubjektive Wende in der Psychoanalyse (S. 7-31). Stuttgart: Klett-Cotta.

Bateman, A., Fonagy, P. (2004). Psychotherapy for borderline personality disorder: Mentalization based treatment. New York, NY: Oxford UP.

Bohleber, W. (2018). Übertragung - Gegenübertragung - Intersubjektivität. Zur Entfaltung ihrer intrinsischen Komplexität. Psyche 72, 702-733. 
Fenichel, O. (1939/2001). Probleme der psychoanalytischen Technik. Giessen: Psychosozial.

Freud, S. (1912b). Zur Dynamik der Übertragung. GWVIII, 364-374.

Freud, S. (1919a). Wege der psychoanalytischen Therapie. GW XII, 183-194.

Freud, S. (1925d). Selbstdarstellung. GW XIV, 31-96.

Green, A. (2002). Die zentrale phobische Position - mit einem Modell der freien Assoziation. Psyche, 56, 409-441.

Heimann, P. (2016 [1950]). Zur Gegenübertragung. In: Dies. Gegenübertragung und andere Schriften zur Psychoanalyse. Vorträge und Aufsätze aus den Jahren 1942-1980 (S. 111-117). Suttgart: Klett-Cotta.

Kernberg, O. F. (2016). New developments in transference focused psychotherapy. Int J Psychoanal 97, 385-407.

Kernberg, O. F. (2014). Liebe und Aggression. Eine unzertrennliche Beziehung. Stuttgart: Schattauer.

Küchenhoff, J. (2013). Zwischen Eröffnung, Vermittlung und Einspruch. Ders. Der Sinn im Nein und die Gabe des Gesprächs. Psychoanalytisches Verstehen zwischen Philosophie und Klinik (S. 327-339). Weilerswist: Velbrück.

Küchenhoff, J. (2005). Psychodynamische Kurz- und Fokaltherapie. Theorie und Praxis. Stuttgart: Schattauer.

Lambert, M. J. (1992). Psychotherapy Outcome Research: Implications for integrative and eclectic therapists. In: Norcross, J. C. \& Goldfried, M. R. (eds.). Handbook of Psychotherapy Integration. New York: Wiley, 94-129.

Sandler, J. (1976). Gegenübertragung und Bereitschaft zur Rollenübernahme. Psyche 30, 297-305.

Sandler J., Sandler A. (1987). The past unconscious, the present unconscious and the vicissitude of guilt. Int J Psychoanal, 68, 331-41.

Ticho, E. (1972). Termination of psychoanalysis: Treatment goals, life goals. Psychoanal Q, 41, 315-33.

Till, W. (2009). Psychoanalytische Aspekte in der Krisenintervention. Psyche, 63, 773-93.

Wampold, B. E., Imel, Z. E. et al. (2018). Die Psychotherapie-Debatte. Was Psychotherapie wirksam macht. Bern: Hogrefe.

Yeomans, F. E., Clarkin, J. F., Kernberg O. F. (2017). Übertragungsfokussierte Psychotherapie für Borderline Patienten. Das TFP-Praxismanual. Stuttgart: Schattauer. 


\section{Angaben zum Autor}

Daniel Sollberger, PD Dr. med. Dr. phil., stv. ärztlicher Direktor Erwachsenenpsychiatrie Baselland, Chefarzt der Zentren für spezifische Psychotherapien und Psychosomatik (ZPP) und für psychosoziale Therapie (ZPS). Studium der Philosophie und Deutschen Literaturwissenschaft in Basel und München, Promotion in Philosophie; Zweitstudium der Humanmedizin in Basel, Facharzt FMH für Psychiatrie und Psychotherapie; Privatdozent an der Medizinischen Fakultät der Universität Basel; TFP-Dozent und Supervisor (ISTFP). 